\title{
Corrigendum: Prevention of Cyclophosphamide-Induced Immunosuppression in Mice With Traditional Chinese Medicine Xuanfei Baidu Decoction
}

\begin{abstract}
Huimin Yan ${ }^{1,2}$, Jia Lu $u^{2,3}$, Jiabao Wang ${ }^{1,2,3}$, Lu Chen $^{1,2,3}$, Yu Wang ${ }^{1,2,3}$, Lin $\operatorname{Li}^{1,2,3}$, Lin Miao $^{1,2,3 *}$ and Han Zhang ${ }^{1,2,3 *}$

'State Key Laboratory of Component-based Chinese Medicine, Tianjin University of Traditional Chinese Medicine, Tianjin, China, ${ }^{2}$ Institute of Traditional Chinese Medicine, Tianjin University of Traditional Chinese Medicine, Tianjin, China, ${ }^{3}$ Key Laboratory of Pharmacology of Traditional Chinese Medical Formulae, Ministry of Education, Tianjin University of Traditional Chinese Medicine, Tianjin, China
\end{abstract}

Keywords: immunosuppression, immune modification, cyclophosphamide, traditional Chinese medicine (TCM), Xuanfei Baidu decoction (XFBD)

\section{A Corrigendum on}

Prevention of Cyclophosphamide-Induced Immunosuppression in Mice With Traditional Chinese Medicine Xuanfei Baidu Decoction

by Yan, H.-M., Lu, J., Wang, J., Chen, L., Wang, Y., Li, L., Miao, L., and Zhang, H. (2021). Front. Pharmacol. 12:730567. doi: 10.3389/fphar.2021.730567

In the original article, there was a mistake in Table $\mathbf{1}$ as published. We would like to correct "Primer sequences used for RT-PCR Table, as errors were introduced in the preparation of this table for publication." The corrected Table 1 appears below.

The authors apologize for this error and state that this does not change the scientific conclusions of the article in any way. The original article has been updated.

TABLE 1 | Primer sequences used for RT-PCR.

This article was submitted to

Ethnopharmacology,

a section of the journal

Frontiers in Pharmacology

Received: 03 November 2021

Accepted: 28 December 2021

Published: 20 January 2022

Citation:

Yan H, Lu J, Wang J, Chen L, Wang Y,

Li L, Miao $L$ and Zhang $H$ (2022)

Corrigendum: Prevention of

Cyclophosphamide-Induced

Immunosuppression in Mice With

Traditional Chinese Medicine Xuanfei

Baidu Decoction.

Front. Pharmacol. 12:808424.

doi: 10.3389/fphar.2021.808424

\begin{tabular}{|c|c|c|}
\hline Gene & Forward primer $\left(5^{\prime}-3^{\prime}\right)$ & Reverse primer $\left(5^{\prime}-3^{\prime}\right)$ \\
\hline IL-2 & AGGAACCTGAAACTCCCCAG & AAATCCAGAACATGCCGCAG \\
\hline IL-4 & TCTCGAATGTACCAGGAGCC & ACCTTGGAAGCCCTACAGAC \\
\hline IL-6 & CTGCAAGAGACTTCCATCCAG & AGTGGTATAGACAGGTCTGTTGG \\
\hline GAPDH & AGGTCGGTGTGAACGGATTTG & TGTAGACCATGTAGTTGAGGTCA \\
\hline
\end{tabular}

Publisher's Note: All claims expressed in this article are solely those of the authors and do not necessarily represent those of their affiliated organizations, or those of the publisher, the editors and the reviewers. Any product that may be evaluated in this article, or claim that may be made by its manufacturer, is not guaranteed or endorsed by the publisher.

Copyright $\odot 2022$ Yan, Lu, Wang, Chen, Wang, Li, Miao and Zhang. This is an open-access article distributed under the terms of the Creative Commons Attribution License (CC BY). The use, distribution or reproduction in other forums is permitted, provided the original author(s) and the copyright owner(s) are credited and that the original publication in this journal is cited, in accordance with accepted academic practice. No use, distribution or reproduction is permitted which does not comply with these terms. 